\title{
O ensino do esporte na educação física escolar: um ensaio sobre as potencialidades do TGFU
}

\section{Sport teaching in school physical education: a essays of TGFU potentialities.}

\section{Enseñanza deportiva en educación física escolar: un ensayos de potencialidades TGFU.}

\author{
iD (9) Rodrigo Márcio de Oliveira e Silva \\ email: rodriggoedfis@gmail.com \\ iD Gustavo De Conti Teixeira Costa \\ Universidade Federal de Goiás, Goiânia, Goiás, Brasil. \\ e-mail: conti02@ufg.br
}

Resumo: O objetivo deste artigo é indicar as potencialidades do Teaching Games for Understanding (TGfU) no ensino do esporte nas aulas de Educação Física. O estudo é um recorte do referencial teórico da dissertação: “Esporte na Educação Física escolar: uma proposta pedagógica no ensino do Handebol", com base em livros, anais de eventos, teses, dissertações e artigos que tematizam o TGfU, escritos em português, inglês e espanhol, sem recorte cronológico, buscando o máximo de obras que contribuíssem com o estudo. São apresentados os princípios pedagógicos, fases e reformulações do TGfU, além de estudos empíricos que evidenciam a capacidade do modelo em incrementar a aprendizagem e a motivação dos alunos, indicando a pertinência do TGfU no ensino do esporte na Educação Física escolar.

Palavras-chave: TGfU. Esporte. Educação Física Escolar.

Abstract: The purpose of this article is to indicate the potential of Teaching Games for Understanfing (TGfU), in the teaching of sport in 
Physical Education classes. The study is an excerpt from the theoretical framework of the dissertation: "Sport in Physical Education at school: a pedagogical proposal in the teaching of Handball", based on books, annals of events, theses, dissertations and articles, which focus on TGfU, written in Portuguese, English and Spanish, without chronological cut, seeking the maximum number of works that contribute to the study. The pedagogical principles, phases and reformulations of the TGfU are presented, as well as empirical studies that demonstrate the model's capacity to increase students' learning and motivation, indicating the relevance of TGfU in the teaching of sport in school Physical Education.

Keywords: TGFU. Sport. School Physical Education.

Resumen: El propósito de este artículo es indicar el potencial de la Enseñanza de Juegos para la Comprensión (TGfU), en la enseñanza del deporte en las clases de Educación Física. El estudio es un extracto del marco teórico de la disertación: “El deporte en la educación física en la escuela: una propuesta pedagógica en la enseñanza del balonmano", basado en libros, anales de eventos, tesis, disertaciones y artículos, que se centran en TGfU, escrito en portugués, inglés y español, sin corte cronológico, buscando el máximo número de obras que contribuyan al estudio. Se presentan los principios pedagógicos, fases y reformulaciones del TGfU, así como estudios empíricos que demuestran la capacidad del modelo para incrementar el aprendizaje y la motivación de los estudiantes, indicando la relevancia del TGfU en la enseñanza del deporte en la Educación Física escolar.

Palabras clave: TGFU. Deporte. Educación Física Escolar.

Submetido em: 27-10-2020

Aceito em: 12-04-2021 
O ensino do esporte na educação física escolar: um ensaio sobre as potencialidades do TGFU Rodrigo Márcio de Oliveira e Silva • Gustavo De Conti Teixeira Costa

\section{Introdução}

A Educação Física escolar brasileira enfrenta diversos problemas que dificultam o desenvolvimento do trabalho pedagógico dos professores e, consequentemente, o aprendizado dos alunos. Dentre os vários desafios enfrentados pelos professores, estão as situações de afastamento e indisciplina nas aulas de Educação Física, fatores que, segundo Darido, González e Ginciene (2018), estão ligados à desmotivação gerada pela forma como os conteúdos são desenvolvidos, pelas situações de insucesso e pela exclusão dos alunos das aulas. Outra problemática da Educação Física escolar brasileira se refere ao ensino do conteúdo esporte (BARROSO, 2018), que se pauta em modelos tradicionais (GIUSTI et al., 2017), com exagerada ênfase no princípio analítico-sintético que se mostra predominante na iniciação esportiva e na Educação Física escolar (ALVES, 2015; CASAGRANDE, 2012)

Segundo Metzler (2011), as abordagens analíticas no ensino de esportes priorizam o desenvolvimento de gestos técnicos e, em menor extensão, das habilidades necessárias para jogar bem o jogo. Dessa forma, a utilização de tais modelos de ensino, descontextualizados da essência tática dos esportes coletivos, não cumpre os pressupostos de representatividade do jogo (CLEMENTE; MENDES, 2011), não estimulam os processos cognitivos que subsidiam a tomada de decisão (GAMA FILHO, 2001) e não incrementarem a motivação dos alunos nas aulas (COSTA; NASCIMENTO, 2004).

A partir da crítica ao modelo tradicional, David Bunker e Rod Thorpe propuseram, em 1982, o modelo TGfU - Teaching Games for Understanding, que altera o foco da aprendizagem para os elementos e problemas táticos do jogo, para o posterior desenvolvimento dos gestos técnicos. Para Graça e Mesquita (2007), no TGfU o jogo deixa de ser visto como um tempo/espaço de aplicação de técnicas e passa a ser percebido como um tempo/espaço de resolução de problemas. 
O ensino do esporte na educação física escolar: um ensaio sobre as potencialidades do TGFU Rodrigo Márcio de Oliveira e Silva • Gustavo De Conti Teixeira Costa

De acordo com Teoldo et al. (2010), o TGfU preconiza e proporciona um maior envolvimento cognitivo dos praticantes nas atividades. Porquanto, o surgimento deste modelo, além de garantir legitimidade ao ensino, oportunizando ao aluno a construção da sua aprendizagem de forma ativa, possibilitou a ocorrência de experiências de sucesso que proporcionaram o incremento de competências na prática do jogo e da motivação pela prática desportiva e nas aulas de Educação Física (HARVEY et al., 2017; HORTIGUELAACALÁ; GARIJO, 2017; GIL-ÁRIAS et al., 2016; LODEWYK; BRACCO, 2018). Deste modo, existem evidências de que este modelo de ensino tem potencial para modificar positivamente a motivação autodeterminada e o comportamento dos alunos nas aulas de Educação Física (VALLS CASTILLO et al., 2017; YAGÜE CABEZÓN; GUTIÉRREZ-GARCÍA, 2018), fato relevante para o ensino dos esportes na atualidade.

Dessa forma, este artigo tem o objetivo de apresentar o modelo TGfU, bem como suas atualizações, indicando potencialidades do modelo no ensino do esporte na Educação Física escolar. Tal fato se justifica pela importância de apresentar aos professores de educação física que tal modelo pode ser adotado em suas respectivas realidades, auxiliando no trato pedagógico do trabalho cotidiano de forma a otimizar o aprendizado dos alunos acerca do conteúdo pretendido, neste caso específico, o esporte.

\section{Princípios pedagógicos do TGfU}

O TGfU prevê o ensino do esporte por meio do desenvolvimento da capacidade de jogo, ou seja, através da sua compreensão tática e sua capacidade de tomada de decisão. Para tal, se utiliza de jogos reduzidos, adaptados ao nível de compreensão e capacidade de ação dos alunos, visando estimular e promover seu melhor entendimento e aprendizagem (KINNERK et al., 2018; WEBB; PEARSON, 2012). As adaptações nos jogos são sustentadas por quatro princípios pedagógicos, sendo: 
O ensino do esporte na educação física escolar: um ensaio sobre as potencialidades do TGFU Rodrigo Márcio de Oliveira e Silva • Gustavo De Conti Teixeira Costa

a. Seleção do tipo de jogo: orienta a atenção do professor para a escolha das atividades de ensino-aprendizagem que possibilitem uma multiplicidade de experiências que condicionem a compreensão de similaridades e diferenças entre as modalidades esportivas (CLEMENTE, 2012). Para tanto, há a necessidade da adoção de um sistema de classificação das modalidades esportivas baseada nas suas características e lógicas internas, como esportes de invasão; esportes de campo e taco; esportes de rede divisória/parede; esportes de combate etc. (THORPE; BUNKER; ALMOND, 1984; GONZALEZ; BRACHT, 2012).

b. Modificação por representação: as atividades propostas devem refletir as características do jogo formal, o que possibilita a compreensão da lógica de funcionamento do esporte que se está ensinando (BUNKER; THORPE, 1986; ALMOND, 2015), potencializando a compreensão tática e as tomadas de decisão, baseadas em situações-problema semelhantes às que se estabelecem no jogo formal (FAGUNDES; RIBAS, 2019).

c. Modificação por exagero: as estruturas didáticas devem ser construídas a partir de alterações nas regras, espaço, tempo de jogo (THORPE; BUNKER; ALMOND, 1984; HOPPER, 2009), visando canalizar a atenção dos alunos para determinado problema tático (GRAÇA; MESQUITA, 2007), objeto de ensino da aula (MCKEEN; WEBB; PEARSON, 2007).

d. Ajustamento da complexidade tática: os problemas táticos e as atividades de ensino-aprendizagem devem ser adequados aos níveis de desenvolvimento e capacidade de intervenção dos alunos (SERRA-OLIVARES; GARCIA-RUBIO, 2017; MORALESBELANDO; ARIAS-ESTERO, 2020).

Complementarmente aos princípios pedagógicos, se faz necessária a utilização de questionamentos, no intuito de estimular a capacidade dos alunos de solucionar os problemas táticos enfatizados na atividade por meio de processos reflexivos internos 
O ensino do esporte na educação física escolar: um ensaio sobre as potencialidades do TGFU Rodrigo Márcio de Oliveira e Silva • Gustavo De Conti Teixeira Costa

(PEARSON; WEBB, 2008). Assim, cabe ao professor facilitar a reflexão, por parte dos alunos, sobre as ações inerentes ao jogo, auxiliando o desenvolvimento de competências que Ihes permitam interpretá-las adequadamente.

\section{Fases do modelo TGfU}

Bunker e Thorpe (1982) estruturam o modelo do TGfU em fases específicas, sistematizadas de forma cíclica e contínua, conforme Figura 1, em que cada etapa apresenta uma caracterização didático-metodológica própria.

Figura 1 - Estruturação TGfU - Bunker e Thorpe (1982)
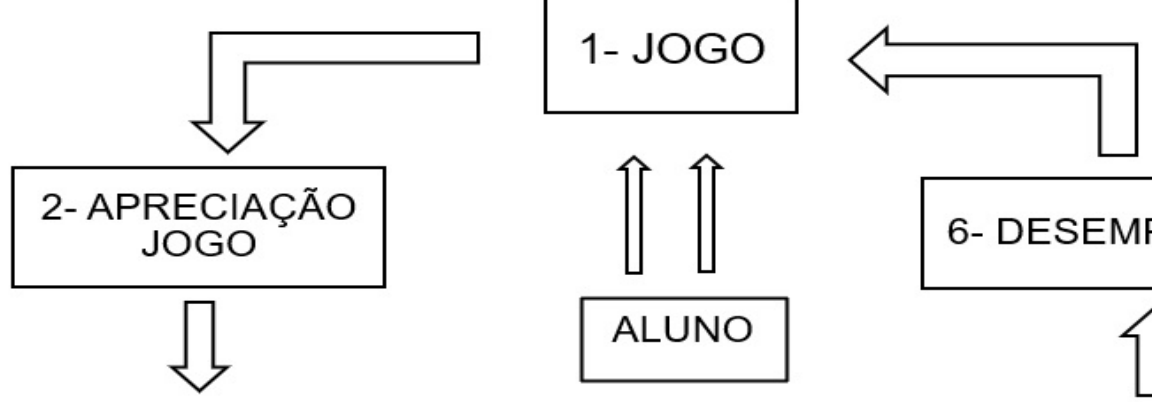

6- DESEMPENHO

3- CONSCIÊNCIA TATICA

5- EXECUÇÃO DA HABILIDADE
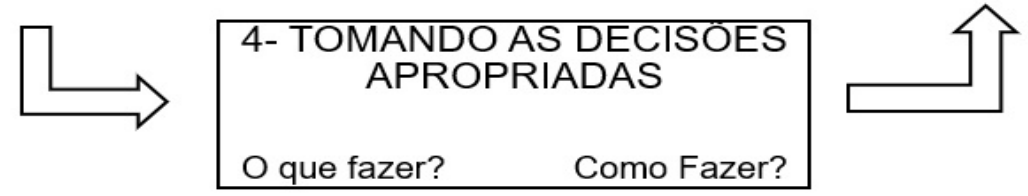

Fonte: Teoldo et al., 2010.

A primeira fase, "Jogo" ou "Forma de Jogo", se refere à seleção de jogos que possibilitem aos alunos uma variada prática esportiva, contemplando diversos problemas táticos, que representem e mantenham a estrutura tática do jogo formal e salientem o comportamento tático a ser desenvolvido na aula (MANDIGO; HOLT, 2002). 
O ensino do esporte na educação física escolar: um ensaio sobre as potencialidades do TGFU Rodrigo Márcio de Oliveira e Silva • Gustavo De Conti Teixeira Costa

A fase "Apreciação do Jogo", envolve aprender as regras da forma de jogo, sua dinâmica e modificações (DUDLEY; BAXTER, 2009). Segundo Bunker e Thorpe (1986), é de suma importância que os alunos compreendam e percebam as regras da forma de jogo, como determinantes das suas estrutura e dinâmica, bem como das técnicas, táticas e habilidades necessárias ao desempenho na mesma.

Na fase "Consciência Tática", propõe-se que, após o entendimento das regras, estrutura e dinâmica do jogo, é necessário considerar as táticas a serem usadas. Nessa fase, os alunos se organizarão estrategicamente para solucionar as tarefas designadas (KIRK; MACPHAIL, 2002).

Na fase "Tomando as Decisões Apropriadas", se estabelece a relação entre o conhecimento declarativo (o que fazer?), desenvolvido na etapa anterior, com o conhecimento processual (como fazer?), diretamente ligado ao domínio da habilidade de execução das ações motoras no contexto de jogo (FAGUNDES, 2019).

Para Bunker e Thorpe (1982), o aperfeiçoamento da execução dos gestos técnicos, só ocorrerá após o aluno conhecer e entender o jogo, bem como suas possibilidades de intervenção e tomada de decisão. Assim, a fase "Execução da Habilidade" enfatiza a apropriação e refinamento dos gestos técnicos, quando esse refinamento for necessário, no auxílio à solução de problemas táticos no contexto do jogo (WEBB; PEARSON; FORREST, 2006; AGUIAR, 2013).

A etapa "Desempenho" é entendida como a avaliação da aprendizagem em relação à compreensão da lógica interna do jogo, à tomada de decisão, à capacidade de percepção e às estratégias e habilidades utilizadas nos contextos específicos dos jogos condicionados (BUNKER; THORPE, 1986).

\section{Propostas de reformulação do TGfU.}

A partir desse modelo inicial, surgiram propostas de modificação do modelo de ensino, destacando-se a de Kirk e Macphail 
O ensino do esporte na educação física escolar: um ensaio sobre as potencialidades do TGFU Rodrigo Márcio de Oliveira e Silva • Gustavo De Conti Teixeira Costa

(2002) e a de Holt, Stream e Bengoechea (2002), que são apresentadas a seguir. A Figura 2, abaixo, reúne a versão original de Bunker e Thorpe (1982), articulada com as propostas de reformulação de Kirk e Macphail (2002) e Holt, Stream e Bengoechea (2002).

Figura 2 - Sobreposição das propostas do Teaching Games for Understanding.

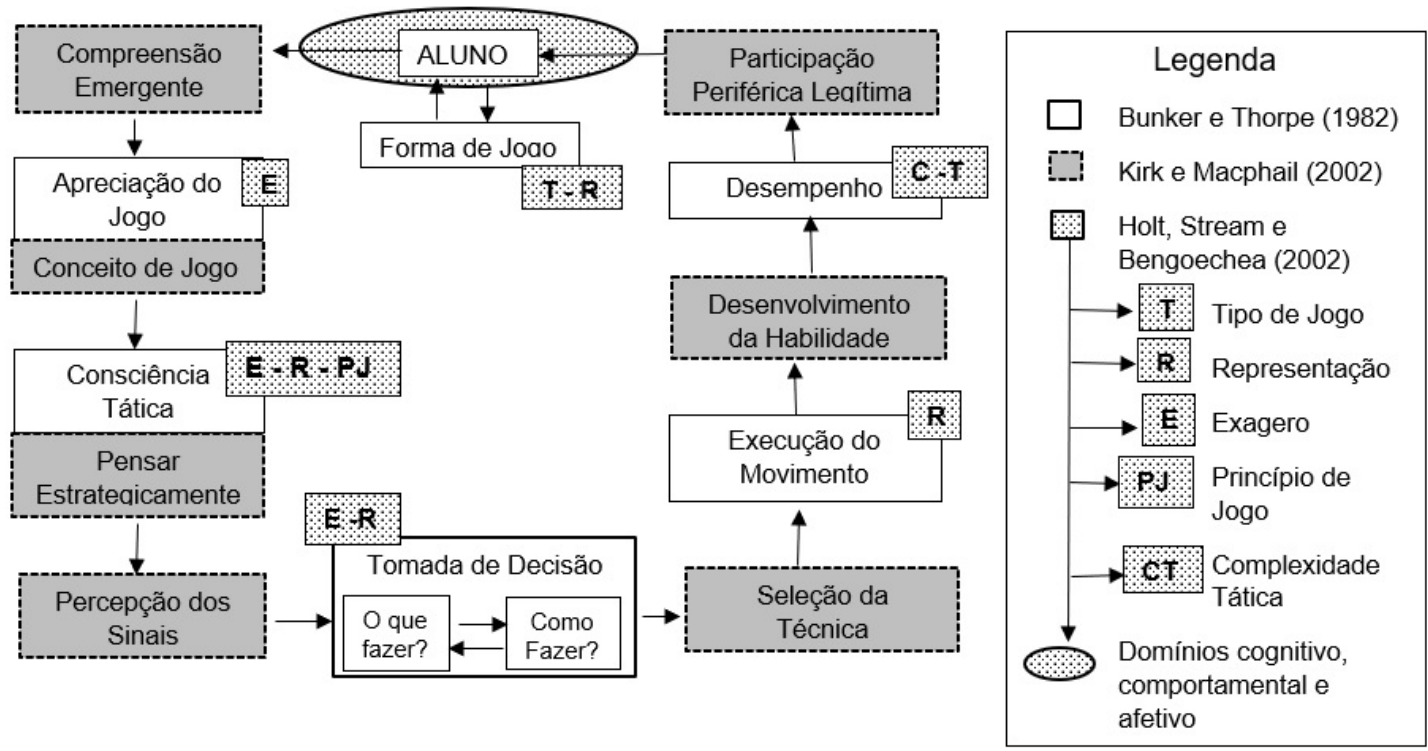

Fonte: Fagundes e Ribas (2019).

\section{Proposta de reformulação de Kirk e Macphail (2002)}

Esses autores entenderam que algumas dimensões do modelo original se encontram omitidas ou subdesenvolvidas e fundamentam a reformulação na Teoria da Aprendizagem Situada, na qual, segundo Lave (1991), a aprendizagem ocorre em função do contexto sociocultural do indivíduo e a partir das interações sociais em que os aprendizes se envolvem em uma comunidade de prática. Neste viés, os autores passam a considerar as dimensões físico-perceptiva, social-interativa e institucional-cultural na aprendizagem. (FAGUNDES; RIBAS, 2019). Neste âmbito, a atualização do modelo TGfU contempla novas dimensões, sendo: 
O ensino do esporte na educação física escolar: um ensaio sobre as potencialidades do TGFU Rodrigo Márcio de Oliveira e Silva • Gustavo De Conti Teixeira Costa

A interfase "Compreensão Emergente" tem a função de denotar a atenção a ser dada em auxiliar os alunos a estabelecerem a ligação entre os propósitos do jogo formal de referência e a sua forma modificada apresentada (TEOLDO et al., 2010; ABURACHID, 2015).

Os elementos "Conceito de Jogo" e "Pensar Estrategicamente", que destacam a ligação entre os conhecimentos declarativo e processual, vão além da transmissão/aquisição de conhecimento das regras e demais aspectos do jogo (GRAÇA; MESQUITA, 2007). Segundo Fagundes e Ribas (2019), a fase "Conceito de Jogo" busca o desenvolvimento do conhecimento dos alunos em relação às regras do jogo, a partir de alterações de elementos da sua lógica interna. Na fase "Pensar Estrategicamente", os alunos assimilarão a lógica interna do jogo condicionado e vão se organizar estrategicamente para solucionar as situações-problema que se apresentarem.

A interfase "Percepção de Sinais", que destaca a necessidade de ajudar os alunos a identificarem os sinais pertinentes (GRAÇA; MESQUITA, 2007), ou seja, saber onde e quais informações buscar no ambiente de jogo, para a adequada tomada de decisão (TEOLDO et al., 2010).

As interfases "Seleção da Técnica" e "Desenvolvimento da Habilidade", que pretendem reforçar a interligação entre a técnica e a táctica, reconhecendo o caráter situado das habilidades e do seu uso estratégico (ABURACHID, 2015). Segundo Kirk e Mcphail (2002), uma habilidade é mais do que uma técnica, correspondendo à capacidade de perceber sinais pertinentes e de executar a técnica em função da tática e da estratégia.

A fase "Desempenho", que é entendida como a avaliação da aprendizagem dos alunos referente à compreensão da lógica interna, às tomadas de decisão, à capacidade de percepção, às estratégias e habilidades utilizadas nos contextos específicos dos jogos condicionados (FAGUNDES, 2019). A interfase "Participação Periférica Legítima" atenta para o significado das experiências de 
O ensino do esporte na educação física escolar: um ensaio sobre as potencialidades do TGFU Rodrigo Márcio de Oliveira e Silva • Gustavo De Conti Teixeira Costa

aprendizagem para os alunos, tendo por referência as práticas extraescolares do esporte enquanto realidades sociais, culturais e institucionais complexas, multifacetadas e heterogêneas (GRAÇA; MESQUITA, 2007).

\section{Proposta de reformulação de Holt, Stream e Bengoe- chea (2002)}

Esta proposta surgiu baseada no pressuposto de que o modelo original não contemplou os quatro princípios pedagógicos do TGfU em todas as suas fases. Dessa forma, os autores propõem um modelo que mantém as seis fases do modelo do TGfU, inserindo os princípios pedagógicos utilizados em cada fase, sendo eles: Amostragem, Modificação por Representação, Modificação por Exagero, Complexidade Tática e Princípios de Jogo.

Os princípios "Amostragem" e "Modificação por Representação", na fase da forma de jogo, auxiliam o alcance do objetivo de introduzir os praticantes em jogos com menor complexidade tática e, gradativamente, modificar as formas e estruturas do jogo, propiciando reflexões sobre os conceitos, conhecimentos e habilidades do jogo. O princípio da "Modificação por Exagero", presente na fase de apreciação do jogo, tem o objetivo de auxiliar o aluno a entender melhor as regras e dinâmica do jogo, por meio da manutenção das regras oficiais e da modificação exagerada de problemas táticos (TEOLDO et al., 2010).

Os "Princípios de Jogo" e as "Modificações por Exagero e Representação", presentes na fase de consciência tática, são importantes para auxiliar os praticantes a compreenderem noções táticas do jogo, basilares para a integração de novos problemas táticos. Segundo Fagundes (2019), no "Princípio de Jogo" é necessário que o professor proporcione aos alunos a consciência da estratégia de atuação da equipe no jogo, além de incentivar o entendimento do papel de cada aluno na estratégia da equipe. 
O ensino do esporte na educação física escolar: um ensaio sobre as potencialidades do TGFU Rodrigo Márcio de Oliveira e Silva • Gustavo De Conti Teixeira Costa

Na fase "Tomada de Decisão", os princípios das Modificações por "Representação" e "Exagero" são importantes na seleção de jogos com maiores níveis de complexidade e na escolha de problemas táticos que possam ser modificados com foco no reconhecimento e nas execuções de soluções táticas (TEOLDO et al., 2010).

Na fase de "Execução Motora", o princípio da "Modificação por Representação" é proposto para dar importância à forma como os praticantes executam as habilidades em contextos que tenham semelhança aos dos jogos. Por fim, na fase "Desempenho", o feedback do professor surge como a forma mais pertinente de repassar ao aluno informações sobre as aprendizagens consumadas ao longo do processo.

\section{TGfU e aprendizagem esportiva: pressupostos sobre o aprendizado e estudos empíricos.}

Segundo Gurvitcha e Metzler (2013), os modelos de ensino são projetados no sentido de promover determinados tipos de aprendizagem e devem ser utilizados de acordo com os objetivos de ensino. Nesse sentido, o TGfU se mostra como modelo de ensino que potencializa o aprendizado esportivo, pois concede primazia ao ensino dos esportes por meio da compreensão tática e dos processos cognitivos de percepção e tomada de decisão (COSTA et al., 2010).

Neste contexto, Metzler (2011) apresenta pressupostos sobre o aprendizado, por meio do TGfU, que auxiliam os professores no ensino dos esportes:

I. a maioria dos alunos acha a participação em jogos mais interessante, motivacional e autêntica do que treinos de desenvolvimento de habilidades que tem pouca aplicação no jogo; 
O ensino do esporte na educação física escolar: um ensaio sobre as potencialidades do TGFU Rodrigo Márcio de Oliveira e Silva • Gustavo De Conti Teixeira Costa

II. alunos podem desenvolver consciência tática e habilidades de tomada de decisão quando esses se tornam objetivo primário da instrução;

III. a consciência tática é pré-requisito para habilidades de desempenho;

IV. consciência tática e tomada de decisão devem ser ensinadas por uma abordagem construtivista, usando uma progressão planejada de atividades de aprendizagem baseadas em problemas táticos;

V. consciência tática e outros tipos de aprendizado do aluno serão transferidos de um esporte para outro, dentro da mesma categoria de classificação.

Estudos sobre este tema mostram que a adoção do TGfU é mais eficaz na prática pedagógica escolar e serão apresentados a seguir.

Morales-Belando, Calderón e Arias-Estero (2019) avaliaram a evolução de 41 alunos na Educação Física escolar, em variáveis relacionadas ao desempenho e aderência após o desenvolvimento de uma unidade didática de floorball (esporte coletivo, disputado em uma quadra, semelhante ao hóquei, mas sem o uso de patins, o objetivo é marcar gols na baliza adversária), alinhada ao TGfU e encontraram como resultados: melhora na tomada de decisão, execução técnica, cobertura, suporte, desempenho do jogo, envolvimento do jogo e em variáveis motivacionais.

Com viés diferente, comparando intervenções baseadas no TGfU com abordagens tradicionais, López, Práxedes e Del Villar (2016), após uma unidade didática de basquetebol, encontraram como resultado: o desenvolvimento maior do conhecimento declarativo no grupo de alunos sujeitos ao ensino pelo TGfU. Nesse mesmo viés, Gouveia et al. (2018), numa unidade de ensino de futebol, handebol e basquetebol, encontraram como resultados: maior tempo de atividade física nas aulas e melhores índices de tomada de decisão no grupo de alunos sujeito ao modelo TGfU. Ao considerar o índice de execução de gestos técnicos em situa- 
O ensino do esporte na educação física escolar: um ensaio sobre as potencialidades do TGFU Rodrigo Márcio de Oliveira e Silva • Gustavo De Conti Teixeira Costa

ção de jogo e performance global, ambos os grupos evoluíram, sem diferença entre as abordagens de ensino. Além disso, Silva (2020), ao comparar o desenvolvimento da aprendizagem tática e técnica em situação de jogo, após uma unidade didática de ensino do handebol, encontrou resultados superiores no grupo de alunos sujeito ao modelo TGfU nos parâmetros passe, dribling, arremessos, ações de ocupação de espaços vazios, penetrações e gols marcados, além das ações defensivas de cobertura e acompanhar o adversário sem posse de bola, em relação ao modelo analítico.

Gil-Árias et al. (2017), ao analisarem a tomada de decisão e a efetividade do ataque no voleibol, a partir de uma unidade didática projetada com base nos princípios metodológicos da pedagogia não linear (caracterizada segundo Chow et al. (2006), pela ênfase na interação entre aluno e meio ambiente e pelo ensino integrado de habilidades técnico-táticas através de jogos modificados) com a manipulação das condicionantes, espaço de jogo, altura da rede e número de participantes, encontraram como resultado a melhora de todos os participantes tanto na tomada de decisão quanto na eficácia da ação de ataque entre as medidas pré e pós-teste. Vale ressaltar a proximidade entre a Pedagogia Não Linear e o TGfU que, segundo Clemente (2012), são modelos ecológicos, ou seja, modelos de ensino que respeitam a integridade e dinâmica do jogo em cada momento da aprendizagem e em cada tarefa implementada, há a proposição de situações semelhantes às do jogo real, centram o processo de ensino no aluno e baseiam tal processo na dinâmica intrínseca dos jogos esportivos. Ainda com base na Pedagogia Não Linear, Práxedes et al. (2019) compararam a diferença de efeito de um programa de treinamento, desenvolvido com base nessa pedagogia e com base no modelo técnico-tradicional, sobre a tomada de decisão e a execução de passe e drible em jogadores de futebol da categoria sub-12, e encontraram como resultados melhoras nas habilidades táticas de criação de linhas de passe, execução do passe e tomada de decisão nos alunos sujeitos à Pedagogia Não Linear, sem diferenças no aprendizado do drible. 
O ensino do esporte na educação física escolar: um ensaio sobre as potencialidades do TGFU Rodrigo Márcio de Oliveira e Silva • Gustavo De Conti Teixeira Costa

Dessa forma, observa-se que o TGfU se apresenta como um modelo de ensino que promove o aprendizado esportivo (GASPARGIL et al., 2019), além de uma ferramenta pedagógica adequada à Educação Física escolar, pois possibilita um aprendizado superior dos alunos em comparação com outros modelos de ensino (ABAD ROBLES et al., 2020; HARVEY; GIL-ARIAS; CLAVER, 2020; ÚBEDA-COLOMER; MONFORTE; DEVIS-DEVIS, 2017). Além disso, constata-se que o modelo TGfU é passível de ser adotado nas aulas de Educação Física e é importante que tal conhecimento seja divulgado aos professores do Brasil, visto o cenário de escassez de trabalhos e estudos científicos relacionados ao ensino do esporte dentro da Educação Física escolar no país (GONÇALVES et al., 2017; MOREIRA et al., 2017; FENSTERSEIFER; SAAD; PEREIRA MORO, 2018; KRAHENBÜHL et al., 2018).

\section{TGfU e motivação nas aulas de Educação Física}

A motivação é um importante fator de intervenção no comportamento humano, influencia de forma significativa o envolvimento dos sujeitos em atividades cotidianas que incluam a aprendizagem, o desempenho e a atenção (PAIM, 2001). No processo de ensino-aprendizagem na Educação Física escolar é essencial que os alunos estejam motivados, uma vez que a motivação estimula mais empenho, persistência e esforço nas atividades (PIRES et al., 2010), o que tende a proporcionar melhor desempenho e aprendizagem (OLIVEIRA, 2018). Ao contrário, a desmotivação impede que os alunos realizem as atividades de forma satisfatória, prejudicando sua aprendizagem, desenvolvimento e fruição dos conteúdos desse componente curricular (TEIXEIRA; MOLETA, 2011; MOREIRA et al., 2017).

Em se tratando do ensino do esporte, é importante o emprego de metodologias de ensino que desenvolvam esse conteúdo de forma sistematizada, planejada e que considerem o nível de desenvolvimento e capacidade de intervenção dos alunos, no sen- 
O ensino do esporte na educação física escolar: um ensaio sobre as potencialidades do TGFU Rodrigo Márcio de Oliveira e Silva • Gustavo De Conti Teixeira Costa

tido de promover maiores e melhores aprendizagens (SANTOS et al., 2015). Em linhas gerais, os estudos nesta temática sugerem que o emprego de modelos pedagógicos alternativos, como abordagens centradas no jogo (HARVEY et al., 2017), influenciam positivamente na motivação e no comportamento dos alunos nas aulas de Educação Física (VALLS CASTILLO et al., 2017; YAGÜE CABEZÓN; GUTIÉRREZ-GARCÍA, 2018).

O modelo TGfU enfatiza o desenvolvimento do conhecimento tático por meio de jogos reduzidos, oportunizando que os alunos sempre "joguem o jogo" à medida que trabalham habilidades técnicas e táticas, o que para a maioria dos alunos é mais interessante, motivacional e autêntico do que treinos de desenvolvimento de habilidades que têm pouca aplicação no jogo (METZLER, 2011). Além disso, Mesquita, Farias e Hastie (2012), apontam que o TGfU, por meio da organização das atividades adaptadas ao nível de habilidades táticas e técnicas dos alunos, oportuniza situações de sucesso e aprendizado aos mesmos. Pires et al. (2010) complementam que tal forma de organização das atividades proporciona incremento na motivação intrínseca dos alunos, o que os direciona ao engajamento, esforço e dedicação ao aprendizado.

Nesse contexto, Hortiguela-Acalá e Garijo (2017) analisaram as percepções dos alunos sobre a motivação e o sucesso (que se relaciona ao desempenho, aprendizagem, controle das habilidades e importância dada à resolução das tarefas) nas aulas de Educação Física, após três unidades esportivas consecutivas (basquetebol, floorball e handebol), comparando as abordagens TGfU e Técnico - Tradicional. Os resultados apontaram que o grupo TGfU apresentou aumento na motivação nas aulas de Educação Física em relação ao grupo da abordagem técnico-tradicional. Úbeda-Colomer, Monforte e Devís-Devís (2017) analisaram as percepções dos alunos numa unidade didática construída com base no TGfU, para o ensino de princípios operacionais segundo Bayer (1994). Como resultado, observou-se o destaque positivo para a forma compreensível como os princípios táticos foram explicados, a forma divertida de se aprender, a oportunidade de participação na cria- 
O ensino do esporte na educação física escolar: um ensaio sobre as potencialidades do TGFU Rodrigo Márcio de Oliveira e Silva • Gustavo De Conti Teixeira Costa

ção dos jogos e a existência de jogos nos quais todos conseguiram desempenho satisfatório.

Sob um enfoque diferente, Lodewyk e Bracco (2018) investigaram, em 25 meninas do $9^{\circ}$ ano de escolaridade, diferenças no sentimento de autoeficácia, no gosto pela atividade física, na ansiedade e as percepções de diferenças entre as experiências anteriores em aulas de Educação Física comparadas às aulas de uma unidade de jogos ministrados por meio do TGfU. Os resultados mostraram que, na comparação com as experiências anteriores nas aulas de Educação Física, as meninas apresentaram maior sentimento de autoeficácia e menores níveis de ansiedade, bem como aumento do sentimento de aprendizagem com destaque para a maneira diferente de ensino-aprendizagem, ou seja, por meio de jogos.

Dessa forma, o TGfU se apresenta como um modelo de ensino que, além de promover o aprendizado esportivo às demandas do aluno, estimula a sua motivação intrínseca (CHEN; DARST, 2002; MORALES-BELANDO; CALDERÓN; ARIAS-ESTERO, 2019, HORTIGUELA-ACALÁ; GARIJO, 2017) e que se configura como um instrumento didático importante no trabalho cotidiano dos professores de Educação Física nas escolas.

\section{Implicações para a prática}

O aprendizado esportivo, nas aulas de Educação Física, se relaciona diretamente à adequada organização das atividades de ensino-aprendizagem, adaptadas aos níveis de conhecimento técnico-tático, capacidade e intervenção dos alunos e direcionadas ao desenvolvimento do aprendizado tático (GRAÇA; MESQUITA, 2007), além de estar relacionado também ao estímulo da motivação desses alunos, oportunizando situações de autonomia e sucesso nas aulas (FIN et al., 2019). Dessa forma, o trabalho dos professores deve se direcionar ao desenvolvimento do conhecimento tático (GRAÇA; MESQUITA, 2007), estimulando o conhecimento e entendimento, por parte dos alunos, de ações importantes do jogo, ca- 
O ensino do esporte na educação física escolar: um ensaio sobre as potencialidades do TGFU Rodrigo Márcio de Oliveira e Silva • Gustavo De Conti Teixeira Costa

pacitando-os a tomar decisões adequadas no seu ambiente dinâmico e imprevisível (FOLLE et al. (2014)). Para tal, deve se utilizar jogos reduzidos, adaptados ao nível de compreensão e capacidade de ação dos alunos, que representem situações semelhantes às situações reais do jogo formal (BUNKER; THORPE, 1986; ALMOND, 2015). Segundo Greco (1998), assim oportuniza-se ao aluno conhecer o jogo, inter-relacionando suas habilidades técnicas, táticas e cognitivas na busca de soluções dos problemas que o jogo propõe.

Especificamente em relação ao ensino dos gestos técnicos, o TGfU preconiza seu ensino e refinamento, quando for necessário, para auxiliar os alunos na solução de problemas táticos no contexto do jogo (WEBB; PEARSON; FORREST, 2006; AGUIAR, 2013). Dessa forma, o ensino da técnica deve ser contextualizado em situações de jogo real (GRAÇA; MESQUITA, 2007), reforçando a interligação entre a técnica e a tática na solução de problemas no ambiente do jogo (KIRK; McPHAIL, 2002).

Dada a relevância da motivação no processo de ensino-aprendizagem, é importante que se busque desenvolver nas aulas atividades que sejam desafiadoras e ao mesmo tempo passíveis de realização satisfatória por parte dos alunos (TAN; CHOW; DAVIDS, 2012). Assim, elas devem oportunizar situações de autonomia e sucesso (FIN et al., 2019), aliadas aos feedbacks positivos do professor (SUN; CHEN, 2010), orientando e/ou incentivando os alunos de forma a favorecer o sentimento de competência dos mesmos. Dessa forma, evitam-se os sentimentos de incompetência, exclusão e desinteresse que podem influenciar negativamente a motivação dos alunos (PAZZINATO DUTRA et al., 2016; SILVA, 2017; OLIVERIA, 2018).

Por fim, é importante destacar que o professor deve adotar um comportamento paciente, com linguagem clara e incentivadora aos alunos (REEVE, 2009), estimulando a participação ativa dos mesmos na construção do seu conhecimento (BARROSO, 2018). Esse estímulo à autonomia direciona os alunos ao melhor desempenho e condiciona a satisfação de necessidades psicológicas de autonomia, competência e pertencimento (JANG; KIM; REEVE, 
O ensino do esporte na educação física escolar: um ensaio sobre as potencialidades do TGFU Rodrigo Márcio de Oliveira e Silva • Gustavo De Conti Teixeira Costa

2012; DA COSTA et al., 2017), proporcionando incremento na motivação intrínseca que direciona o aluno ao engajamento, esforço e dedicação ao aprendizado (PIRES et al., 2010).

\section{Considerações Finais}

Observa-se, na Educação Física escolar brasileira, diversos desafios que dificultam o melhor desenvolvimento do trabalho dos professores e, consequentemente, o aprendizado dos alunos. Neste contexto, é necessário difundir concepções contemporâneas de ensino que possibilitem maior interação dos alunos com os conteúdos da Educação Física. Para tal, o processo de ensino-aprendizagem deve estimular a prática do aluno, uma vez que a motivação oportuniza maiores níveis de empenho e envolvimento nas atividades, resultando em maiores níveis de aprendizagem.

A partir do que foi discutido neste artigo, observa-se que o modelo TGfU promove maior motivação dos alunos nas aulas de Educação Física, possibilitando a sua interação com o ensino tático-técnico. Desta forma, esse modelo de ensino, promove estímulo na motivação intrínseca dos alunos a partir de duas características da estruturação das atividades: o ajustamento das tarefas de ensino-aprendizagem às habilidades táticas e técnicas, e capacidade de intervenção dos alunos. Esta adequação no ensino favorece situações de sucesso e aprendizado e, pelo desenvolvimento das atividades com foco nos alunos, direciona-os para a solução dos problemas táticos dos jogos, o que promove sua participação ativa na construção do seu conhecimento, favorecendo sua autonomia. Ao se tratar do ensino do esporte nas aulas de Educação Física, é importante que os objetivos no processo de ensino-aprendizagem estejam relacionados ao conhecimento e entendimento, por parte dos alunos, das ações importantes do jogo, ao desenvolvimento das capacidades de selecionar e executar respostas adequadas no ambiente do jogo, de tomada de decisão e, por consequência, do aprendizado esportivo. 
O ensino do esporte na educação física escolar: um ensaio sobre as potencialidades do TGFU Rodrigo Márcio de Oliveira e Silva • Gustavo De Conti Teixeira Costa

Dessa forma, o TGfU, ao preconizar o ensino do esporte por meio da compreensão tática e dos processos cognitivos de percepção e tomada de decisão, promove o desenvolvimento de habilidades técnicas de forma contextualizada a situações semelhantes às de jogo real e do conhecimento tático, facilitando o processo de tomada de decisão, necessários para o desempenho competente no jogo. Além disso, cabe ressaltar que no contexto da Educação Física escolar, no qual há uma multiplicidade esportes a serem ensinados, além de outras manifestações da cultura corporal, o ensino por meio do TGfU tem a possibilidade de promover a transferência de habilidades táticas, favorecendo o aprendizado de outra modalidade esportiva na mesma categorização.

\section{Referências}

ABAD ROBLES, M. T.; COLLADO-MATEO, D.; FERNÁNDEZESPÍNOLA, C.; CASTILLO VIERA, E.; GIMÉNEZ FUENTES-GUERRA, F. J. Effects of Teaching Games on Decision Making and Skill Execution: A Systematic Review and MetaAnalysis. International Journal Environ Res Public Health. v. 17(2): 505. 2020. doi: 10.3390/ijerph17020505.

ABURACHID, L. M. C. Impacto de diferentes métodos de ensino no desempenho tático-técnico no Badminton. 2015. (Tese de Doutorado). Programa de Pós-Graduação em Educação Física. Universidade Federal de Minas Gerais. Belo Horizonte. 2015.

AGUIAR, B. C. Relatório de estágio pedagógico desenvolvido na escola básica $2^{\circ}$ e $3^{\circ}$ ciclos Dra. Maria Alica Gouveia junto da turma B do $7^{\circ}$ ano no ano letivo 2012-2013: a problemática da aplicação prática do modelo Teaching Games for Understanding (TGfU) no ensino básico na EB 2,3 Dra. Maria Alice Gouveia. Dissertação (Mestrado). Universidade de Coimbra. Coimbra, 2013. ALMOND, L. Rethinking Teaching Games For Understanding. Ágora, para la Educación Fisica y el Deporte, v. 17, n. 1, p. 1525, 2015. 
O ensino do esporte na educação física escolar: um ensaio sobre as potencialidades do TGFU Rodrigo Márcio de Oliveira e Silva • Gustavo De Conti Teixeira Costa

ALVES, D. M. Métodos de ensino da Educação Física escolar utilizados pelos professores da rede privada da cidade de Pelotas-RS. Revista Científica Da Faculdade De Educação E Meio Ambiente, 6(1), 109-122, 2015. https://doi.org/10.31072/rcf. v6i1.277

BARROSO, A. L. R. Inquietações no tratamento do esporte na Educação Física escolar. 2018. Disponível em: https://edutec. unesp.br/moodle/pluginfile.php/138422/mod_resource/content/1/te xto_para_impressao.pdf. Acesso em: 15 fev. 2020.

BAYER, C. 0 ensino dos desportos colectivos. Lisboa: Dinalivros, 1994.

BUNKER, D.; THORPE, R. A model for the teaching of games in secondary schools. Bulletin of Physical Education, v. 18, n. 1, p. 5-8, 1982.

BUNKER, D.; THORPE, R. The curriculum model. In: Thorpe, R; Bunker, D.; Almond, L (Ed.), Rethinking games teaching. ( $p$. 7-10). 1986.

CASAGRANDE, C. G. Ensino e aprendizagem dos esportes coletivos: análise dos métodos de ensino na cidade de UberlândiaMG. Dissertação de Mestrado. Programa Pós-Graduação em Educação Física. UFTM - Universidade Federal do Triângulo Mineiro. 2012.

CHEN, A.; DARST, P. W. Individual and situational interest: The role of gender and skill. Contemporary Educational Psychology, v. 27(2), p. 250-269. 2002.

CLEMENTE, F. M. Princípios Pedagógicos dos Teaching Games for Understanding e da Pedagogia Não-Linear no Ensino da Educação Física. Movimento, Rio Grande do Sul, v. 18, n. 2, p. 315-335, abr./ jun. 2012.

CLEMENTE, F.; MENDES, R. Aprender o jogo jogando: uma abordagem transdisciplinar. Revista Científica Exedra, v. 5, n. 1, p. 27-36, 2011 
O ensino do esporte na educação física escolar: um ensaio sobre as potencialidades do TGFU Rodrigo Márcio de Oliveira e Silva • Gustavo De Conti Teixeira Costa

COSTA, I. T; GARGANTA, J. M.; GRECO, P. J.; COSTA, V. Estrutura temporal e métodos de ensino em jogos desportivos coletivos. Revista Palestra, v. 10, n. x, p. 26-33, 2010.

COSTA, L. C. A.; NASCIMENTO, J. V. O ensino da técnica e da tática: novas abordagens metodológicas. Revista da Educação Física UEM. Maringá, v. 15, n. 2, p. 49-56. 2004.

DA COSTA, L. C. A.; PASSOS, P. C. B.; SOUZA, V. F. M.; VIEIRA, L. F. Educaçao Física e esportes: Motivando para a prática cotidiana escolar. Revista Movimento, Porto Alegre, v. 23, n. 3, p. 935-948, jul./set. de 2017.

DARIDO, S.C.; GONZALEZ F. J.; GINCIENE, G. O afastamento e a indisciplina dos alunos nas aulas de Educação Física Escolar. 2018. Disponível em: https://edutec.unesp.br/moodle/pluginfile. php/138421/mod_resource/content/1/texto_para_impressao.pdf. Acesso em: 18 jan. 2019.

DUDLEY, D.; BAXTER, D. Assessing levels of student understanding in pre-service teachers using a two-cycle SOLO model. AsiaPacific Journal of Teacher Education v. 37, n. 3, 283-293. 2009. DOI: 10.1080/13598660903052282.

FAGUNDES, F. M. 0 modelo Teaching Games for Understanding e a Praxiologia Motriz: Sistematização do Ensino para compreensão da Lógica interna do Voleibol. 2019. (Dissertação de mestrado). Programa de Pós-Graduação em Educação Física. Universidade Federal de Santa Maria-RS. 2019.

FAGUNDES, F. M.; RIBAS, J. F. M. Ensino dos Esportes para Compreensão da Lógica Interna: Aproximações do Teaching Games For Understanding com a Visão Praxiológica. Revista Acción Motriz, v. 23, p. 109-119, 2019.

FENSTERSEIFER, A.; SAAD, M.; PEREIRA MORO, A. Futebol: Uma Investigação do estado do conhecimento das dissertações e teses produzidas no Brasil. Pensar a Prática, 21(2). 2018. https://doi. org/10.5216/rpp.v21i2.44088. 
O ensino do esporte na educação física escolar: um ensaio sobre as potencialidades do TGFU Rodrigo Márcio de Oliveira e Silva • Gustavo De Conti Teixeira Costa

FIN, G.; MORENO-MÚRCIA, J. A.; BARETA, E.; NODARI JÚNIOR, R. J. Estilo interpessoal docente e desmotivação na educação física: validação das escalas no contexto brasileiro. Revista Brasileira de Ciências do Esporte, Porto Alegre, v. 41, n. 4, p. 427-436, out. 2019. Disponível em: https://doi.org/10.1016/j.rbce.2018.07.005. Acesso em: 18 fev. 2020.

FOLLE, A.; QUINAUD, R. T.; BARROSO, M L. C.; ROCHA, J. C. S.; RAMOS, V.; NASCIMENTO, J. V. Construção e validação preliminar de instrumento de avaliação 256 do desempenho técnico-tático individual no basquetebol. Revista de Educação Física, v. 25, p. 405-418, 2014.

GAMA FILHO, J. G. Metodologia do Treinamento Técnico no Futebol. In: GARCIA, E. S.; LEMOS, K. L. M. (org). Temas Atuais VI em Educação Física e esportes. Belo Horizonte. Heath. 2001 p. 86-106.

GASPAR-GIL, V. M.; ÁLVAREZ, F. D. V.; PIZARRO, A. P.; DOMÍNGUEZ, A. M. El Cuestionamento como Herramienta Fundamental para el Desarrollo de la Toma de Decisiones de los Alunos em Educación Física. Revista Movimento, Porto Alegre, v. 25, 2019.

GIL-ARIAS A.; HARVEY, S.; CÁRCELES, A.; PRÁXEDES, A.; DEL VILLAR, F. Impact of a hybrid TGfU-Sport Education unit on student motivation in physical education. PLoS One 12(6): 2017. e0179876. https://doi.org/10.1371/journal.pone.0179876.

GIL-ÁRIAS, A.; ARROYO, M. P. M.; RABAZ, F. C.; DOMÍNGUEZ, A. M.; ÁLVAREZ, F. D. V. Manipulación de los condicionantes de la tarea en Educación Física: Una propuesta desde la pedagogía no lineal. Retos, n. 29, p. 22-27. 2016.

GIUSTI, J. G. M., GALATTI, L. R., DA CUNHA VOSER, R., AZEVEDO, M. R. O Ensino do Esporte através do jogo: Análise, possibilidades e desafios na Educação Física escolar. Pensar a Prática, 20(3) 2017. https://doi.org/10.5216/rpp.v20i3.40709

GONÇALVES, L. F.; ROJO, J. R.; COVICHIOLLI, F. R.; SILVA, M. M. Mapeamento da produção do conhecimento sobre a modalidade 
O ensino do esporte na educação física escolar: um ensaio sobre as potencialidades do TGFU Rodrigo Márcio de Oliveira e Silva • Gustavo De Conti Teixeira Costa

do basquetebol nos periódicos brasileiros. Pensar a prática, Goiânia, v. 20, n. 3, p. 461-475, 2017.

GONZÁLEZ, F. J.; BRACHT, V. Metodologia do Ensino dos Esportes Coletivos. Vitória: UFES, 2012.

GOUVEIA, E. R.; IHLE, A; GOUVEIA, B.; KLIEGEL, M.; MALHO, H.; FREITAS, B.; OLIVERIA, R.; GASPAR, M. FREITAS, D.; PRUDENE, J.; LOPES. H. O ensino dos Jogos Desportivos Coletivos de Invasão segundo uma Abordagem Tática ao Jogo: um estudo quasi-experimental em alunos do Ensino Básico. In: Didática da Educação Física: perspetivas, interrogações e alternativas. Universidade da Madeira. p. 74-86, 2018. ISBN: 978-989-8805-23-2.

GRAÇA, A.; MESQUITA, I. A investigação sobre os modelos de ensino dos jogos esportivos coletivos. Revista Portuguesa de Ciências do Desporto, Porto, v. 7, n. 3, p. 401-421, 2007.

GRECO, P. J. Iniciação Esportiva Universal: metodologia da iniciação esportiva na escola e no clube. Belo Horizonte: Editora UFMG. v. 2, 1998.

GURVITCHA, R.; METZLER, M. Aligning Learning Activities with Instructional Models. Journal of PhysicalEducation, Recreation \& Dance, 84:3, 30-37. 2013. 263 Disponível em: http://dx.doi.org/ 10.1080/07303084.2013.767719. Acesso em: 15 dez. 2019.

HARVEY, S.; GIL-ÁRIAS, A.; CLAVER, F. Effects of teaching games for understanding on tactical knowledge development in middle school physical education. Journal of Physical Education and Sport, v. 20 (3), Art 189, p. 1369 - 1379, 2020 online ISSN: 2247 806X; p-ISSN: 2247 - 8051; ISSN - L = 2247 - 8051 @ JPES. HARVEY, S.; GIL-ÁRIAS, A.; SMITH, M. L.; SMITH, L. R. Middle and Elementary School Students' Changes in Self-Determined Motivation in a Basketball Unit Taught using the Tactical Games Model. Journal of Human Kinetics, v. 59, p. 39-53, 2017. DOI: 10.1515/hukin-2017-0146.

HOLT, N.; STREAM, W.; BENGOECHEA, E. Expanding the teaching games for understanding model: new avenues for future research 
O ensino do esporte na educação física escolar: um ensaio sobre as potencialidades do TGFU Rodrigo Márcio de Oliveira e Silva • Gustavo De Conti Teixeira Costa

and practice. Journal of Teaching in Physical Education, v. 21, p. 162-176, 2002.

HOPPER, T. Game-as-teacher in TGfU and Video-games: Enabling constraints in learning through game-play. In: 26th ACHPER International Conference. Brisbane, Australia. 2009. HORTIGUELA-ACALÁ, D.; GARIJO, A. H. Teaching Games for Understanding: a Comprehensive Approach to Promote Student's Motivation in Physical Education - Journal of Human Kinetics, v. 59. p. 17-27, 2017.

JANG. H; KIM, E. J.; REEVE, J. Longitudinal test of self-determination theory's motivation mediation. Journal of Educational Psychology, v.104(4), p. 175-188. 2012.

KINNERK, P.; HARVEY, S.; MACDONNCHA, C.; LYONS, M. A Review of the Game Based Approaches to Coaching Literature in Competitive Team Sport Settings. Quest, v. 70, p. 1-18, 2018. KIRK, D.; MACPHAIL, A. Teaching Games for Understanding and Situated Learning: Rethinking the Bunker-Thorpe Model. Journal of Teaching in Physical Education, v. 21 (2), p.177-192. Jan., 2002.

KRAHENBÜHL, T.; ROSA, M. L. R.; AMAZONAS, S. M. F.; RODRIGUES, H. A.; LEONARDO, L. Produção Científica sobre o Ensino do Handebol na Educação Física Escolar. Corpoconsciência, Cuiabá, v. 22, n. 03, p. 74-85, set./dez., 2018. LAVE, J. Cognición en la práctica. Barcelona: Paidós, 1991. LODEWYK K.; BRACCO E. Differences in Adaptive Outcomes between Previous Physical Education and a Teaching Games for Understanding Games Unit in Adolescent Girls. Journal of Research, v. 10, n. 1, p. 12-20, 2018.

LÓPEZ, I.; PRÁXEDES, A.; DEL VILLAR. Effect of an Intervention Teaching Program, based on TGFU Model, on the Cognitive and Execution Variables, in the Physical Education Context. European Journal of Human Movement, 37, 88- 108, 2016. 
O ensino do esporte na educação física escolar: um ensaio sobre as potencialidades do TGFU Rodrigo Márcio de Oliveira e Silva • Gustavo De Conti Teixeira Costa

MANDIGO, J.; HOLT, N. The inclusion of optimal challenge in teaching games for understanding. Physical and Health Education Journal, v. (66)3, p. 14-21. 2002.

MCKEEN, K.; WEBB, P. I.; PEARSON, P. J. Promoting physical activity through teaching games for understanding in undergraduate teacher education. In: DINIZ, J. A. (Eds.), AIESEP 2005 World Congress, Lisboa, p. 251-258. 2007.

MESQUITA, I.; FARIAS, C.; HASTIE, P. The impact of a hybrid Sport Education- Invasion Games Competence Model soccer unit on students' decision making, skill execution and overall game performance. European Physical Education Review, v. 18(2), p. 205-219. 2012.

METZLER, M. W. Instructional models for Physical Education. $3^{a}$ Edição. Scottsdale: Holcomb Hathaway, 2011.

MORALES-BELANDO, M. T.; ARIAS-ESTERO, J. L. Adaptación práctica del enfoque Teaching Games for Understanding para la enseñanza de la vela en iniciación. Retos, v. 37, p. 738-741. 2020. MORALES-BELANDO, M. T.; CALDERÓN, A.; ARIAS-ESTERO, J. L. Improvement in game performance and adherence after an aligned TGfU floorball unit in physical. Physical Education and Sport Pedagogy, v. 23:6, p. 657-671, 2019. DOI: 10.1080/17408989.2018.1530747.

MORALES-BELANDO, M. T.; CALDERÓN, A.; ARIAS-ESTERO, J. L. Improvement in game performance and adherence after an aligned TGfU floorball unit in physical. Physical Education and Sport Pedagogy, v. 23:6, p. 657-671, 2019. DOI: 10.1080/17408989.2018.1530747.

MOREIRA, C. H.; MACIEL, L. F. P.; NASCIMENTO, R. K.; FOLLE, A. Motivação de Estudantes nas aulas de Educação Física: um estudo de Revisão. Corpoconsciência, Cuiabá, v. 21, n. 02, p. 67-79, 2017.

OLIVEIRA, E. H. Motivação nas aulas de Educação Física: perspectiva dos alunos do ensino fundamental. 2018. Dissertação 
O ensino do esporte na educação física escolar: um ensaio sobre as potencialidades do TGFU Rodrigo Márcio de Oliveira e Silva • Gustavo De Conti Teixeira Costa

(Mestrado). Faculdade de Filosofia Ciências e Letras de Ribeirão Preto. Universidade de São Paulo. Ribeirão Preto/SP. 2018.

PAIM, M. C. C. Fatores motivacionais e desempenho no futebol.

Revista da Educação Física, v. 12, n. 2, p. 73-79, 2001.

PAZZINATO DUTRA, R.; VALERIO, M.; GOULART, A. S.; CASTRO, C. F.; LOPES, C. D.; FIUSSEN, C.; GONÇALVES, C.; PIASSAROLLO, D.; MASIERO, G.; QUADROS, L.; LANAU, R. M.; SCAGLIONI, R.; VALERO, V. M. Desmotivação nas aulas de Educação Física, segundo relatos de estudantes do $9^{\circ}$ ano do ensino fundamental. Revista Didática Sistêmica, v. 18, n. 1, p. 70-78, jul. 2016. ISSN 18093108. Disponível em: https://periodicos.furg.br/redsis/article/ view/6518. Acesso em: 18 fev. 2020.

PEARSON, P.; WEBB, P. Developing effective questioning in Teaching Games for Understanding (TGfU). In: 1st Asia Pacific Sport in Education Conference: Ngunyawaiendi Yerthoappendi Play to Educate, Adelaide, 2008.

PIRES, A.; CID, L.; BORREGO, C.; ALVES, J.; SILVA, C. Validação preliminar de um questionário para avaliar as necessidades psicológicas básicas em Educação Física. Motricidade, v. 6, n. 1, 2010.

PRÁXEDES, A.; DEL VILLAR, F. A.; MORENO, A.; GIL-ARIAS, A.; DAVIDS, K. Effects of a nonlinear pedagogy intervention programme on the emergent tactical behaviours of youth footballers. Physical Education and Sport Pedagogy, v. 24 n. 4 p. 332-343 2019 DOI: $10.1080 / 17408989.2019 .1580689$.

REEVE J. Why teachers adopt a controlling motivating style toward students and how they can become more autonomy supportive. Educational Psychologist, v. 44(3), p. 159-177. 2009.

SANTOS, C. R.; SILVA, C. C.; DAMASCENO, M. L.; MEDINA-PAPST, J.; MARQUES, I. Efeito da atividade esportiva sistematizada sobre o desenvolvimento motor de crianças de sete a 10 anos. Rev. bras. educ. fís. esporte [online], v. 29, n. 3, p. 497-506, 2015. ISSN 1807-5509. https://doi.org/10.1590/1807- 55092015000300497. 
O ensino do esporte na educação física escolar: um ensaio sobre as potencialidades do TGFU Rodrigo Márcio de Oliveira e Silva • Gustavo De Conti Teixeira Costa

SERRA-OLIVARES, J.; GARCIA-RUBIO, J. La problemática táctica, clave en el diseño representativo de tareas desde el enfoque de la pedagogía na lineal aplicada al deporte. RETOS, n. 32, p. 270278, 2017.

SILVA, R. M. O. Esporte na Educação Física escolar: uma proposta pedagógica no ensino do handebol. Dissertação (Mestrado) - Universidade Federal de Goiás, Goiânia, 2020.

SILVA, T. F. R. Conhecimento declarativo técnico-tático no handebol entre estudantes. 2013. Dissertação (Mestrado) Universidade Estadual Paulista. Bauru, 2017.

SUN H.; CHEN A. F. A pedagogical understanding of the self determination theory in physical education. Quest, v. 62, n. 4, p. 364384, Nov. 2010.

TAN, C. W. K.; CHOW, J. Y.; DAVIDS, K. How does TGfU work?': examining the relationship between learning design in TGfU and a nonlinear pedagogy. Physical Education and Sport Pedagogy, v. 17(4), p. 331-348. 2012.

TEIXEIRA, F. A.; MOLETA, A. F. Motivação nas aulas de Educação Física. Anais do X Encontro nacional de Educação - EDUCERE. Pontifícia Universidade Católica do Parana- PUC, Curitiba, 2011. TEOLDO, I.; GRECO, P. J.; MESQUITA, I.; GRAÇA, A.; GARGANTA, J. O Teaching Games For Understanding (TGfU) Como Modelo De Ensino Dos Jogos Desportivos Coletivos. Revista Palestra, v. 10, p. 69-77, 2010.

THORPE, R. D.; BUNKER, D. J.; ALMOND, L. A Change In The Focus Of Teaching Games. In: PIÉRON, M.; GRAHAM, G. (Eds.), Sport pedagogy: Olympic Scientific Congress proceedings, Book 6, $1^{\text {a }}$ Ed. Eugene. Champaign, IL: Human Kinetics. p. 163-169, 1984.

ÚBEDA-COLMER, J.; MONFORTE, J.; DEVIS-DEVIS, J. Percepción del alumnado sobre una Unidad Didáctica de enseñanza comprensiva de los juegos deportivos de invasión en Educación Física. Retos, v. 31, p. 275-281. 2017. 
O ensino do esporte na educação física escolar: um ensaio sobre as potencialidades do TGFU Rodrigo Márcio de Oliveira e Silva • Gustavo De Conti Teixeira Costa

VALLS CASTILLO, A.; BARTOLL, O. C.; PERIS, C. C. Aprendizaje comprensivocooperativo del balonmano en Educación Física: efecto sobre el clima motivacional. E-Balonmano.com: revista de Ciencias del Deporte, v. 13, n. 1, p. 53-66, 2017.

WEBB, P. I.; PEARSON, P. J. Creative unit and lesson planning through a thematic/integrated approach to Teaching Games for Understanding (TGfU). New Zealand Physical Educator, v. 45 (3), p. 17-22. 2012.

WEBB, P.; PEARSON, P.; FORREST, G. Teaching Games For Understanding (TGfU) In Primary And Secondary Physical Education. In: International Conference for Health, Physical Education, Recreation, Sport and Dance. 1st Oceanic Congress Wellington, New Zealand, 2006.

YAGÜE CABEZÓN, J. M. Y.; GUTIÉRREZ-GARCÍA, C. Aprendizaje del fútbol en la educación física escolar a partir de un modelo comprensivo. Revista Española de Educación Física y Deportes, v. 423, p. 41-55. 2018.

\section{Agradecimentos}

Agradecemos ao Ministério da Ciência, Tecnologia, Inovação e Comunicações (MCTIC) e Conselho Nacional de Desenvolvimento Científico e Tecnológico (CNPq) que financiaram este artigo por meio da Chamada Universal MCTIC/CNPq n. 29/2018.

\section{Publisher}

Universidade Federal de Goiás. Faculdade de Educação Física e Dança. Publicação no Portal de Periódicos UFG. As ideias expressadas neste artigo são de responsabilidade de seus autores, não representando, necessariamente, a opinião dos editores ou da universidade. 